\title{
Splitting methods for a convex feasibility problem in Hilbert spaces
}

\author{
Yunpeng Zhang ${ }^{\mathrm{a}}$, Yanling $\mathrm{Li}^{\mathrm{b}, *}$ \\ ${ }^{a}$ College of Electric Power, North China University of Water Resources and Electric Power, Zhengzhou, 450011, China. \\ ${ }^{b}$ School of Mathematics and Information Sciences, North China University of Water Resources and Electric Power University, \\ Zhengzhou 450011, China.
}

Communicated by R. Saadati

\begin{abstract}
In this paper, we investigate a convex feasibility problem based on a splitting method. Strong convergence theorems are established without the aid of metric projections in the framework of real Hilbert spaces. (C)2016 All rights reserved.
\end{abstract}

Keywords: Equilibrium problem, monotone operator, feasibility problem, Hilbert space, projection. 2010 MSC: 47H05, 90C33.

\section{Introduction}

A very interesting problem in diverse areas of physics and mathematics consists of trying to find a special solution. This problem is referred to as the convex feasibility problem. It can be described as follows: $D_{1}, D_{2}, \cdots, D_{N}$, where $N$ denotes some positive integer, are finitely many closed convex nonempty subsets of a Hilbert space with $D:=\cap_{i=1}^{N} D_{i} \neq \emptyset$. Convex feasibility problem is to find a solution in $D$. Closely related subjects of the problem are variational inequality problems, zero point problems, fixed point problems and equilibrium problem; see [1, 3, 4, 17, 8, 9, 10, 11, 12, 13, 14, 15, 17, 18, 19, 20, 23, 24, 26, 27, 28, 29, 30] and the references therein.

Many problems of convex programming can be reduced to that of finding a zero point $x$ of a maximal monotone operator $B$ on a Hilbert space $H$; see [21, 22, 26]. A fundamental technique for solving a zero point equation involving a monotone operator is the proximal point algorithm. The resolvent $J_{s}=(I+$

\footnotetext{
${ }^{*}$ Corresponding author

Email address: zhangypliyl@yeah.net (Yanling Li)
} 
$s B)^{-1}$, where $I$ stands for the identity mapping and $s$ is some positive real number has many important properties that make it a central tool in monotone operator theory and its applications. Especially, it is firmly nonexpansive. In the context of monotone operator theory, what is known as the Douglas-Rachford algorithm is a splitting scheme initially proposed in [12] for finding a zero of the sum of two monotone operators. Splitting algorithms for problems involving the sum of two monotone operators give some applications to the obstacle problems and minimization problems. In this paper, we investigate a convex feasibility problem based on a splitting method. Strong convergence theorems are established without the aid of metric projections in the framework of real Hilbert spaces.

\section{Preliminaries}

Let $H$ be a real Hilbert space with the inner product $\langle\cdot, \cdot\rangle$ and the norm $\|\cdot\|$ and let $C$ be a nonempty closed convex subset of $H$.

Let $S: C \rightarrow C$ be a mapping. $F(S)$ stands for the fixed point set of $S$. Recall that $S$ is said to be contractive iff there exits a constant $\alpha \in(0,1)$ such that

$$
\|S x-S y\| \leq \alpha\|x-y\|, \quad \forall x, y \in C .
$$

$S$ is said to be nonexpansive iff

$$
\|S x-S y\| \leq\|x-y\|, \quad \forall x, y \in C .
$$

$S$ is said to be firmly nonexpansive iff $\|S x-S y\|^{2} \leq\langle S x-S y, x-y\rangle ; S$ is said to be strictly pseudocontractive iff there exists a constant $\kappa \in[0,1)$ such that

$$
\|S x-S y\|^{2} \leq\|x-y\|^{2}+\kappa\|(x-S x)-(y-S y)\|^{2}, \quad \forall x, y \in C .
$$

The class of strictly pseudocontractive mappings was introduced by Browder and Petryshyn [6]. It is clear that nonexpansive mappings are strictly pseudocontractive mappings with $\kappa=0$.

Let $F: C \times C \rightarrow \mathbb{R}$ be a bifunction, where $\mathbb{R}$ denotes the set of real numbers. Consider the following equilibrium problem in the terminology of Blum and Oettli [5]

$$
\text { Find } x \in C \text { such that } F(x, y) \geq 0, \quad \forall y \in C \text {. }
$$

In this paper, the solution set of problem $(2.1)$ is denoted by $E P(F)$.

To study equilibrium problem (2.1), we may assume that $F$ satisfies the following conditions:

(A1) $F(x, x)=0$ for all $x \in C$;

(A2) $F$ is monotone, i.e., $F(x, y)+F(y, x) \leq 0$ for all $x, y \in C$;

(A3) for each $x, y, z \in C$, $\lim \sup _{t \downarrow 0} F(t z+(1-t) x, y) \leq F(x, y)$;

(A4) for each $x \in C, y \mapsto F(x, y)$ is convex and lower semi-continuous.

Let $A: C \rightarrow H$ be a mapping. Recall that $A$ is said to be monotone iff

$$
\langle A x-A y, x-y\rangle \geq 0, \quad \forall x, y \in C .
$$

$A$ is said to be strongly monotone iff there exists a constant $\alpha>0$ such that

$$
\langle A x-A y, x-y\rangle \geq \alpha\|x-y\|^{2}, \quad \forall x, y \in C .
$$

$A$ is said to be inverse-strongly monotone iff there exists a constant $\alpha>0$ such that

$$
\langle A x-A y, x-y\rangle \geq \alpha\|A x-A y\|^{2}, \quad \forall x, y \in C .
$$


It is clear that $A$ is inverse-strongly monotone if and only if the inverse of $A$ is strongly monotone.

A set-valued mapping $B: H \rightarrow 2^{H}$ is said to be monotone if for all $x, y \in H, f \in B x$ and $g \in B y$ imply $\langle x-y, f-g\rangle \geq 0$. A monotone mapping $B: H \rightarrow 2^{H}$ is maximal if the graph $G(B)$ of $B$ is not properly contained in the graph of any other monotone mapping. It is known that a monotone mapping $B$ is maximal if and only if, for any $(x, f) \in H \times H,\langle x-y, f-g\rangle \geq 0$ for all $(y, g) \in G(B)$ implies $f \in B x$. Next, we use $D(B)$ to denote the domain of $B$. If $B$ is maximal monotone, we may define a single-valued operator $J_{r}=(I+r B)^{-1}: H \rightarrow H$, where $r$ is some positive constant. The single-valued operator is called the resolvent of $B$ for the constant $r$.

In order to prove our main results, we also need the following lemmas.

Lemma 2.1 ([2]). Let $C$ be a closed convex subset of a real Hilbert space $H$ and let $A$ be a maximal monotone operator on $H$. For any $\lambda>0$ and $\mu>0$, we have $J_{\lambda} x=J_{\mu}\left(\frac{\mu}{\lambda} x+\left(1-\frac{\mu}{\lambda}\right) J_{\lambda} x\right)$, where $J_{\lambda}=(I+\lambda A)^{-1}$ and $J_{\mu}=(I+\mu A)^{-1}$.

Lemma $2.2([6])$. Let $C$ be a closed convex subset of a real Hilbert space $H$ and let $S: C \rightarrow C$ be a strictly pseudocontractive mapping. Then $I-S$ is demiclosed at zero.

Lemma $2.3([5])$. Let $C$ be a closed convex subset of a real Hilbert space $H$ and let $F: C \times C \rightarrow \mathbb{R}$ be a bifunction satisfying $(A 1)-(A 4)$. Then, for any $r>0$ and $x \in H$, there exists $z \in C$ such that

$$
r F(z, y)+\langle y-z, z-x\rangle \geq 0, \quad \forall y \in C .
$$

Further, define

$$
T_{r} x=\{z \in C: r F(z, y)+\langle y-z, z-x\rangle \geq 0, \quad \forall y \in C\}
$$

for all $r>0$ and $x \in H$. Then, the following hold:

(a) $T_{r}$ is single-valued;

(b) $\left\|T_{r} x-T_{r} y\right\|^{2} \leq\left\langle T_{r} x-T_{r} y, x-y\right\rangle$;

(c) $F\left(T_{r}\right)=E P(F)$;

(d) $E P(F)$ is closed and convex.

Lemma 2.4 ([6]). Let $C$ be a closed convex subset of a real Hilbert space $H$ and let $S: C \rightarrow H$ be a strictly pseudocontractive mapping with the constant $\kappa$. Define a mapping $T$ by $T=\delta I+(1-\delta) S$, where $\delta$ is a constant in $[0,1]$. If $\delta \in[\kappa, 1)$ then $T$ is nonexpansive with $F(T)=F(S)$.

Lemma $2.5([25])$. Let $\left\{x_{n}\right\}$ and $\left\{y_{n}\right\}$ be bounded sequences in a Hilbert space $H$ and let $\left\{\beta_{n}\right\}$ be a sequence in $(0,1)$ with $0<\liminf _{n \rightarrow \infty} \beta_{n} \leq \limsup _{n \rightarrow \infty} \beta_{n}<1$. Suppose $x_{n+1}=\left(1-\beta_{n}\right) y_{n}+\beta_{n} x_{n}$ for all integers $n \geq 0$ and

$$
\limsup _{n \rightarrow \infty}\left(\left\|y_{n+1}-y_{n}\right\|-\left\|x_{n+1}-x_{n}\right\|\right) \leq 0 .
$$

Then $\lim _{n \rightarrow \infty}\left\|y_{n}-x_{n}\right\|=0$.

Lemma 2.6 ([16]). Assume that $\left\{\alpha_{n}\right\}$ is a sequence of nonnegative real numbers such that $\alpha_{n+1} \leq(1-$ $\left.\gamma_{n}\right) \alpha_{n}+\delta_{n}+e_{n}$, where $\left\{\gamma_{n}\right\}$ is a sequence in $(0,1),\left\{e_{n}\right\}$ and $\left\{\delta_{n}\right\}$ are sequences such that

(i) $\sum_{n=1}^{\infty} \gamma_{n}=\infty, \sum_{n=1}^{\infty} e_{n}<\infty$;

(ii) $\limsup _{n \rightarrow \infty} \delta_{n} / \gamma_{n} \leq 0$ or $\sum_{n=1}^{\infty}\left|\delta_{n}\right|<\infty$.

Then $\lim _{n \rightarrow \infty} \alpha_{n}=0$.

Lemma 2.7 ([3]). Let $C$ be a closed convex subset of a real Hilbert space $H$. Let $A: C \rightarrow H$ be a mapping and let $B$ be a maximal monotone operator on $H$. Then $F\left(J_{s}(I-s A)\right)=(A+B)^{-1}(0)$, where $J_{s}=(I+s B)^{-1}$. 


\section{Main results}

Theorem 3.1. Let $C$ be a closed convex subset of a real Hilbert space $H$. Let $A: C \rightarrow H$ be an inversestrongly monotone mapping with the positive constant $\alpha$ and let $B$ be a maximal monotone operator such that $D(B) \subset C$. Let $F$ be a bifunction from $C \times C$ to $\mathbb{R}$ which satisfies (A1)-(A4). Let $S: C \rightarrow H$ be a strictly pseudocontractive mapping with the constant $\kappa \in[0,1)$ and let $f$ be a contractive mapping on $H$ with the constant $\beta \in(0,1)$. Assume that $\Omega=F(S) \cap(A+B)^{-1}(0) \cap E P(F)$ is nonempty. Let $\left\{r_{n}\right\}$ and $\left\{s_{n}\right\}$ be positive real number sequences. Let $\left\{\alpha_{n}\right\},\left\{\beta_{n}\right\},\left\{\gamma_{n}\right\}$ and $\left\{\delta_{n}\right\}$ be real number sequences in $(0,1)$ such that $\alpha_{n}+\beta_{n}+\gamma_{n}=1$. Let $\left\{x_{n}\right\}$ be a sequence generated in the process: $x_{1} \in H, y_{n}=\left(I+s_{n} B\right)^{-1}\left(z_{n}-s_{n} A z_{n}+e_{n}\right)$, $x_{n+1}=\alpha_{n} f\left(x_{n}\right)+\beta_{n} x_{n}+\gamma_{n} \delta_{n} y_{n}+\left(1-\delta_{n}\right) \gamma_{n} S y_{n}$, where $\left\{e_{n}\right\}$ is a sequence in $H$ and $\left\{z_{n}\right\}$ is a sequence in $C$ such that $F\left(z_{n}, z\right)+\frac{1}{r_{n}}\left\langle z-z_{n}, z_{n}-x_{n}\right\rangle \geq 0, \forall z \in C$. Assume that the control sequences satisfy the following conditions: $\lim _{n \rightarrow \infty} \alpha_{n}=0$ and $\sum_{n=1}^{\infty} \alpha_{n}=\infty ; 0<\liminf _{n \rightarrow \infty} \beta_{n} \leq \limsup _{n \rightarrow \infty} \beta_{n}<1$; $\sum_{n=1}^{\infty}\left\|e_{n}\right\|<\infty, \lim _{n \rightarrow \infty}\left|\delta_{n+1}-\delta_{n}\right|=0$ and $\kappa \leq \delta_{n} \leq \delta<1 ; \lim _{n \rightarrow \infty}\left|r_{n+1}-r_{n}\right|=0$ and $\liminf _{n \rightarrow \infty} r_{n}>0$; $\lim _{n \rightarrow \infty}\left|s_{n+1}-s_{n}\right|=0$, and $0<s \leq s_{n} \leq s^{\prime}<2 \alpha$, where $\delta, s, s^{\prime}$ are real constants. Then $\left\{x_{n}\right\}$ converges strongly to $q=P_{\Omega} f(q)$.

Proof. By using the conditions imposed on $\left\{s_{n}\right\}$, we find that

$$
\left\|\left(I-s_{n} A\right) x-\left(I-s_{n} A\right) y\right\|^{2} \leq\|x-y\|^{2}-s_{n}\left(2 \alpha-s_{n}\right)\|A x-A y\|^{2} .
$$

This implies that $I-s_{n} A$ is nonexpansive. Put $J_{s_{n}}=\left(I+s_{n} B\right)^{-1}$ and $S_{n}=\delta_{n} I+\left(1-\delta_{n}\right) S$. It follows from Lemma 2.4 that $S_{n}$ is nonexpansive with $F\left(S_{n}\right)=F(S)$ and $J_{s_{n}}$ is firmly nonexpansive. Letting $p \in \Omega$ be fixed arbitrarily, we have

$$
\begin{aligned}
\left\|y_{n}-p\right\| & \leq\left\|\left(z_{n}-s_{n} A z_{n}+e_{n}\right)-\left(p-s_{n} A p\right)\right\| \\
& \leq\left\|T_{r_{n}} x_{n}-p\right\|+\left\|e_{n}\right\| \\
& \leq\left\|x_{n}-p\right\|+\left\|e_{n}\right\|
\end{aligned}
$$

It follows that

$$
\begin{aligned}
\left\|x_{n+1}-p\right\| & \leq \alpha_{n}\left\|f\left(x_{n}\right)-p\right\|+\beta_{n}\left\|x_{n}-p\right\|+\gamma_{n}\left\|S_{n} y_{n}-p\right\| \\
& \leq \alpha_{n} \beta\left\|x_{n}-p\right\|+\alpha_{n}\|f(p)-p\|+\beta_{n}\left\|x_{n}-p\right\|+\gamma_{n}\left\|y_{n}-p\right\| \\
& \leq \alpha_{n} \beta\left\|x_{n}-p\right\|+\alpha_{n}\|f(p)-p\|+\beta_{n}\left\|x_{n}-p\right\|+\gamma_{n}\left(\left\|x_{n}-p\right\|+\left\|e_{n}\right\|\right) \\
& \leq\left(1-\alpha_{n}(1-\beta)\right)\left\|x_{n}-p\right\|+\alpha_{n}\|f(p)-p\|+\left\|e_{n}\right\| \\
& \leq \max \left\{\left\|x_{n}-p\right\|, \frac{\|f(p)-p\|}{1-\beta}\right\}+\left\|e_{n}\right\| .
\end{aligned}
$$

It follows that

$$
\left\|x_{n}-p\right\| \leq \max \left\{\left\|x_{1}-p\right\|, \frac{\|f(p)-p\|}{1-\beta}\right\}+\sum_{n=1}^{\infty}\left\|e_{n}\right\| .
$$

This shows that $\left\{x_{n}\right\}$ is bounded. Since the mapping $P_{\Omega} f$ is contractive, there exists an unique fixed point. Next, we denote the unique fixed point by $q$. Now, we are in a position to show $\lim \sup _{n \rightarrow \infty}\left\langle f(q)-q, x_{n}-q\right\rangle \leq$ 0 . To show it, we can choose a subsequence $\left\{x_{n_{i}}\right\}$ of $\left\{x_{n}\right\}$ such that

$$
\limsup _{n \rightarrow \infty}\left\langle f(q)-q, x_{n}-q\right\rangle=\lim _{i \rightarrow \infty}\left\langle f(q)-q, x_{n_{i}}-q\right\rangle
$$

Since $\left\{x_{n_{i}}\right\}$ is bounded, we can choose a subsequence $\left\{x_{n_{i_{j}}}\right\}$ of $\left\{x_{n_{i}}\right\}$ which converges weakly some point $x$. We may assume, without loss of generality, that $\left\{x_{n_{i}}\right\}$ converges weakly to $x$.

In view of $z_{n}=T_{r_{n}} x_{n}$, one has $F\left(z_{n}, z\right)+\frac{1}{r_{n}}\left\langle z-z_{n}, z_{n}-x_{n}\right\rangle \geq 0, \forall z \in C$ and $F\left(z_{n+1}, z\right)+\frac{1}{r_{n+1}}\langle z-$ $\left.z_{n+1}, z_{n+1}-x_{n+1}\right\rangle \geq 0, \forall z \in C$. Hence, one has $F\left(z_{n}, z_{n+1}\right)+\frac{1}{r_{n}}\left\langle z_{n+1}-z_{n}, z_{n}-x_{n}\right\rangle \geq 0$ and $F\left(z_{n+1}, z_{n}\right)+$ 
$\frac{1}{r_{n+1}}\left\langle z_{n}-z_{n+1}, z_{n+1}-x_{n+1}\right\rangle \geq 0, \forall z \in C$. From the monotonicity of $F$, one has $\left\langle z_{n+1}-z_{n}, \frac{z_{n}-x_{n}}{r_{n}}-\right.$ $\left.\frac{z_{n+1}-x_{n+1}}{r_{n+1}}\right\rangle \geq 0$. It follows that

$$
\left\|z_{n+1}-z_{n}\right\| \leq\left\|x_{n+1}-x_{n}\right\|+\frac{\left|r_{n+1}-r_{n}\right|}{r_{n+1}}\left\|T_{r_{n+1}} x_{n}-x_{n+1}\right\| .
$$

Putting $\rho_{n}=z_{n}-s_{n} A z_{n}+e_{n}$, we find that

$$
\begin{aligned}
\left\|\rho_{n+1}-\rho_{n}\right\| & \leq\left\|z_{n+1}-z_{n}\right\|+\left\|A z_{n}\right\|\left|s_{n+1}-s_{n}\right|+\left\|e_{n+1}-e_{n}\right\| \\
& \leq\left\|x_{n+1}-x_{n}\right\|+\frac{\left|r_{n+1}-r_{n}\right|}{r_{n+1}}\left\|T_{r_{n+1}} x_{n}-x_{n+1}\right\|+\left\|A z_{n}\right\|\left|s_{n+1}-s_{n}\right|+\left\|e_{n+1}-e_{n}\right\| .
\end{aligned}
$$

On the other hand, we find from Lemma 2.1 that

$$
\begin{aligned}
\left\|y_{n+1}-y_{n}\right\|= & \left\|J_{s_{n}}\left(\frac{s_{n}}{s_{n+1}} \rho_{n+1}+\left(1-\frac{s_{n}}{s_{n+1}}\right) J_{s_{n+1}} \rho_{n+1}\right)-J_{s_{n}} \rho_{n}\right\| \\
\leq & \left\|\frac{s_{n}}{s_{n+1}}\left(\rho_{n+1}-\rho_{n}\right)+\left(1-\frac{s_{n}}{s_{n+1}}\right)\left(J_{s_{n+1}} \rho_{n+1}-\rho_{n}\right)\right\| \\
\leq & \left\|\rho_{n+1}-\rho_{n}\right\|+\frac{\left|s_{n+1}-s_{n}\right|}{s}\left\|J_{s_{n+1}} \rho_{n+1}-\rho_{n+1}\right\| \\
\leq & \left\|x_{n+1}-x_{n}\right\|+\frac{\left|r_{n+1}-r_{n}\right|}{r_{n+1}}\left\|T_{r_{n+1}} x_{n}-x_{n+1}\right\|+\left\|A z_{n}\right\|\left|s_{n+1}-s_{n}\right| \\
& +\left\|e_{n+1}-e_{n}\right\|+\frac{\left|s_{n+1}-s_{n}\right|}{s}\left\|J_{s_{n+1}} \rho_{n+1}-\rho_{n+1}\right\| .
\end{aligned}
$$

Putting $\lambda_{n}=\frac{x_{n+1}-\beta_{n} x_{n}}{1-\beta_{n}}$, we have

$$
\begin{aligned}
\left\|\lambda_{n+1}-\lambda_{n}\right\| \leq & \frac{\alpha_{n+1}\left\|f\left(x_{n+1}\right)-S_{n+1} y_{n+1}\right\|}{1-\beta_{n+1}}+\frac{\alpha_{n}\left\|f\left(x_{n}\right)-S_{n} y_{n}\right\|}{1-\beta_{n}} \\
& +\left\|S_{n+1} y_{n+1}-S_{n} y_{n}\right\| \\
\leq & \frac{\alpha_{n+1}\left\|f\left(x_{n+1}\right)-S_{n+1} y_{n+1}\right\|}{1-\beta_{n+1}}+\frac{\alpha_{n}\left\|f\left(x_{n}\right)-S_{n} y_{n}\right\|}{1-\beta_{n}} \\
& +\left\|y_{n+1}-y_{n}\right\|+\left|\delta_{n+1}-\delta_{n}\right|\left\|y_{n}-S y_{n}\right\| .
\end{aligned}
$$

Combining (3.1) with 3.2 finds that

$$
\begin{aligned}
\left\|\lambda_{n+1}-\lambda_{n}\right\|-\left\|x_{n+1}-x_{n}\right\| \leq & \frac{\alpha_{n+1}\left\|f\left(x_{n+1}\right)-S_{n+1} y_{n+1}\right\|}{1-\beta_{n+1}}+\frac{\alpha_{n}\left\|f\left(x_{n}\right)-S_{n} y_{n}\right\|}{1-\beta_{n}} \\
& +\frac{\left|r_{n+1}-r_{n}\right|}{r_{n+1}}\left\|T_{r_{n+1}} x_{n}-x_{n+1}\right\|+\left\|A z_{n}\right\|\left|s_{n+1}-s_{n}\right| \\
& +\left\|e_{n+1}-e_{n}\right\|+\frac{\left|s_{n+1}-s_{n}\right|}{s}\left\|J_{s_{n+1}} \rho_{n+1}-\rho_{n+1}\right\| \\
& +\left|\delta_{n+1}-\delta_{n}\right|\left\|y_{n}-S y_{n}\right\| .
\end{aligned}
$$

By using conditions imposed on the control sequences, one has

$$
\limsup _{n \rightarrow \infty}\left(\left\|\lambda_{n+1}-\lambda_{n}\right\|-\left\|x_{n+1}-x_{n}\right\|\right) \leq 0 .
$$

It follows from Lemma 2.5 that $\lim _{n \rightarrow \infty}\left\|\lambda_{n}-x_{n}\right\|=0$, which in turn implies that

$$
\lim _{n \rightarrow \infty}\left\|x_{n+1}-x_{n}\right\|=0
$$


Since $T_{r_{n}}$ is firmly nonexpansive, we find that

$$
\begin{aligned}
\left\|z_{n}-p\right\|^{2} & =\left\|T_{r_{n}} x_{n}-T_{r_{n}} p\right\|^{2} \\
& \leq \frac{1}{2}\left(\left\|x_{n}-p\right\|^{2}+\left\|z_{n}-p\right\|^{2}-\left\|x_{n}-z_{n}\right\|^{2}\right) .
\end{aligned}
$$

That is, $\left\|z_{n}-p\right\|^{2} \leq\left\|x_{n}-p\right\|^{2}-\left\|x_{n}-z_{n}\right\|^{2}$. It follows that

$$
\begin{aligned}
\left\|x_{n+1}-p\right\|^{2} & \leq \alpha_{n}\left\|f\left(x_{n}\right)-p\right\|^{2}+\beta_{n}\left\|x_{n}-p\right\|^{2}+\gamma_{n}\left\|S_{n} y_{n}-p\right\|^{2} \\
& \leq \alpha_{n}\left\|f\left(x_{n}\right)-p\right\|^{2}+\beta_{n}\left\|x_{n}-p\right\|^{2}+\gamma_{n}\left\|y_{n}-p\right\|^{2} \\
& \leq \alpha_{n}\left\|f\left(x_{n}\right)-p\right\|^{2}+\beta_{n}\left\|x_{n}-p\right\|^{2}+\gamma_{n}\left(\left\|z_{n}-p\right\|^{2}+\left\|e_{n}\right\|\left(\left\|e_{n}\right\|+2\left\|z_{n}-p\right\|\right)\right) \\
& \leq \alpha_{n}\left\|f\left(x_{n}\right)-p\right\|^{2}+\left\|x_{n}-p\right\|^{2}-\gamma_{n}\left\|x_{n}-z_{n}\right\|^{2}+\left\|e_{n}\right\|\left(\left\|e_{n}\right\|+2\left\|z_{n}-p\right\|\right),
\end{aligned}
$$

which implies that

$$
\begin{aligned}
\gamma_{n}\left\|x_{n}-z_{n}\right\|^{2} \leq & \alpha_{n}\left\|f\left(x_{n}\right)-p\right\|^{2}+\left(\left\|x_{n}-p\right\|+\left\|x_{n+1}-p\right\|\right)\left\|x_{n}-x_{n+1}\right\| \\
& +\left\|e_{n}\right\|\left(\left\|e_{n}\right\|+2\left\|z_{n}-p\right\|\right) .
\end{aligned}
$$

By using conditions imposed on the control sequences, we find from (3.3) that

$$
\lim _{n \rightarrow \infty}\left\|z_{n}-x_{n}\right\|=0
$$

Hence, $\left\{z_{n_{i}}\right\}$ converges weakly to $x \in C$.

Next, we show $x \in E P(F)$. Notice that

$$
F\left(z_{n}, z\right)+\frac{1}{r_{n}}\left\langle z-z_{n}, z_{n}-x_{n}\right\rangle \geq 0, \quad \forall z \in C .
$$

By using the monotonicity of $F$, we see that $\frac{1}{r_{n}}\left\langle z-z_{n}, z_{n}-x_{n}\right\rangle \geq F\left(z, z_{n}\right), \forall z \in C$. Replacing $n$ by $n_{i}$, we arrive at $\left\langle z-z_{n_{i}}, \frac{z_{n_{i}}-x_{n_{i}}}{r_{n_{i}}}\right\rangle \geq F\left(z, z_{n_{i}}\right), \forall z \in C$. It follows from (3.4) that $0 \geq F(z, x)$. For each $t$ with $0<t \leq 1$, let $z_{t}=t z+(1-t) x$, where $z \in C$. It follows that $z_{t} \in C$. Hence, we have hence $F\left(z_{t}, x\right) \leq 0$. It follows that

$$
0=F\left(z_{t}, z_{t}\right) \leq t F\left(z_{t}, z\right)+(1-t) F\left(z_{t}, x\right) \leq t F\left(z_{t}, z\right),
$$

which yields that $F\left(z_{t}, z\right) \geq 0, \forall z \in C$. Letting $t \downarrow 0$, we obtain that $F(x, z) \geq 0, \forall z \in C$. This implies that $x \in E P(F)$.

Since $A$ is inverse-strongly monotone, we have

$$
\begin{aligned}
\left\|x_{n+1}-p\right\|^{2} \leq & \alpha_{n}\left\|f\left(x_{n}\right)-p\right\|^{2}+\beta_{n}\left\|x_{n}-p\right\|^{2}+\gamma_{n}\left\|S_{n} y_{n}-p\right\|^{2}, \\
\leq & \alpha_{n}\left\|f\left(x_{n}\right)-p\right\|^{2}+\beta_{n}\left\|x_{n}-p\right\|^{2}+\gamma_{n}\left(\left\|\left(z_{n}-s_{n} A z_{n}\right)-\left(p-s_{n} A p\right)\right\|^{2}\right. \\
& \left.+\left\|e_{n}\right\|\left(\left\|e_{n}\right\|+2\left\|z_{n}-p\right\|\right)\right) \\
\leq & \alpha_{n}\left\|f\left(x_{n}\right)-p\right\|^{2}+\beta_{n}\left\|x_{n}-p\right\|^{2}+\gamma_{n}\left(\left\|z_{n}-p\right\|^{2}-s_{n}\left(2 \alpha-s_{n}\right)\left\|A z_{n}-A p\right\|^{2}\right. \\
& \left.+\left\|e_{n}\right\|\left(\left\|e_{n}\right\|+2\left\|z_{n}-p\right\|\right)\right) \\
\leq & \alpha_{n}\left\|f\left(x_{n}\right)-p\right\|^{2}+\left\|x_{n}-p\right\|^{2}-s_{n}\left(2 \alpha-s_{n}\right) \gamma_{n}\left\|A z_{n}-A p\right\|^{2} \\
& +\left\|e_{n}\right\|\left(\left\|e_{n}\right\|+2\left\|z_{n}-p\right\|\right) .
\end{aligned}
$$

This yields that

$$
\begin{aligned}
s_{n}\left(2 \alpha-s_{n}\right) \gamma_{n}\left\|A z_{n}-A p\right\|^{2} \leq & \alpha_{n}\left\|f\left(x_{n}\right)-p\right\|^{2}+\left(\left\|x_{n}-p\right\|^{2}+\left\|x_{n+1}-p\right\|\right)\left\|x_{n+1}-x_{n}\right\| \\
& +\left\|e_{n}\right\|\left(\left\|e_{n}\right\|+2\left\|z_{n}-p\right\|\right) .
\end{aligned}
$$


In view of conditions imposed on the control sequences that

$$
\lim _{n \rightarrow \infty}\left\|A z_{n}-A p\right\|=0 .
$$

Since $J_{s_{n}}$ is firmly nonexpansive, one has

$$
\begin{aligned}
\left\|y_{n}-p\right\|^{2} \leq & \left\langle\left(I-s_{n} A\right) z_{n}+e_{n}-\left(I-s_{n} A\right) p, y_{n}-p\right\rangle \\
= & \frac{1}{2}\left\{\left\|\left(I-s_{n} A\right) z_{n}+e_{n}-\left(I-s_{n} A\right) p\right\|^{2}+\left\|y_{n}-p\right\|^{2}\right. \\
& \left.-\left\|\left(I-s_{n} A\right) z_{n}+e_{n}-\left(I-s_{n} A\right) p-\left(y_{n}-p\right)\right\|^{2}\right\} \\
\leq & \frac{1}{2}\left\{\left\|z_{n}-p\right\|^{2}+L_{n}+\left\|y_{n}-p\right\|^{2}-\left\|z_{n}-y_{n}-\left(s_{n}\left(A z_{n}-A p\right)-e_{n}\right)\right\|^{2}\right\} \\
\leq & \frac{1}{2}\left\{\left\|x_{n}-p\right\|^{2}+L_{n}+\left\|y_{n}-p\right\|^{2}-\left\|z_{n}-y_{n}\right\|^{2}\right. \\
& \left.+2\left\|z_{n}-y_{n}\right\|\left\|s_{n}\left(A z_{n}-A p\right)-e_{n}\right\|-\left\|s_{n}\left(A z_{n}-A p\right)-e_{n}\right\|^{2}\right\},
\end{aligned}
$$

where $L_{n}=\left\|e_{n}\right\|\left(\left\|e_{n}\right\|+2\left\|z_{n}-p\right\|\right)$. This yields that

$$
\begin{aligned}
\left\|y_{n}-p\right\|^{2} \leq & \left\|x_{n}-p\right\|^{2}+L_{n}-\left\|z_{n}-y_{n}\right\|^{2}+2 s_{n}\left\|z_{n}-y_{n}\right\|\left\|A z_{n}-A p\right\| \\
& +2\left\|z_{n}-y_{n}\right\|\left\|e_{n}\right\| .
\end{aligned}
$$

Therefore, we have

$$
\begin{aligned}
\left\|x_{n+1}-p\right\|^{2} \leq & \alpha_{n}\left\|f\left(x_{n}\right)-p\right\|^{2}+\beta_{n}\left\|x_{n}-p\right\|^{2}+\gamma_{n}\left\|S_{n} y_{n}-p\right\|^{2} \\
\leq & \alpha_{n}\left\|f\left(x_{n}\right)-p\right\|^{2}+\beta_{n}\left\|x_{n}-p\right\|^{2}+\gamma_{n}\left\|y_{n}-p\right\|^{2} \\
\leq & \alpha_{n}\left\|f\left(x_{n}\right)-p\right\|^{2}+\left\|x_{n}-p\right\|^{2}+L_{n}-\gamma_{n}\left\|z_{n}-y_{n}\right\|^{2} \\
& +2 s_{n} \gamma_{n}\left\|z_{n}-y_{n}\right\|\left\|A z_{n}-A p\right\|+2\left\|z_{n}-y_{n}\right\|\left\|e_{n}\right\| .
\end{aligned}
$$

It follows that

$$
\begin{aligned}
\gamma_{n}\left\|z_{n}-y_{n}\right\|^{2} \leq & \alpha_{n}\left\|f\left(x_{n}\right)-p\right\|^{2}+\left(\left\|x_{n}-p\right\|+\left\|x_{n+1}-p\right\|\right)\left\|x_{n}-x_{n+1}\right\|+L_{n} \\
& +2 s_{n} \gamma_{n}\left\|z_{n}-y_{n}\right\|\left\|A z_{n}-A p\right\|+2\left\|z_{n}-y_{n}\right\|\left\|e_{n}\right\| .
\end{aligned}
$$

By using (3.3) and 3.5), we find that

$$
\lim _{n \rightarrow \infty}\left\|z_{n}-y_{n}\right\|=0
$$

Hence, $\left\{y_{n_{i}}\right\}$ converges weakly to $x \in C$.

Now, we show that $x \in(A+B)^{-1}(0)$. In view of $y_{n}=J_{s_{n}}\left(z_{n}-s_{n} A z_{n}+e_{n}\right)$, one has $\frac{z_{n}-y_{n}+e_{n}}{s_{n}}-A z_{n} \in B y_{n}$. Since $B$ is maximal monotone, we get, for any $(\mu, \nu) \in B$,

$$
\left\langle y_{n}-\mu, \frac{z_{n}-y_{n}+e_{n}}{s_{n}}-A z_{n}-\nu\right\rangle \geq 0 .
$$

Replacing $n$ by $n_{i}$ and letting $i \rightarrow \infty$, we get from (3.6) that

$$
\langle x-\mu,-A x-\nu\rangle \geq 0 .
$$

This gives $-A x \in B x$, that is, $0 \in(A+B)(x)$. This show that $x \in(A+B)^{-1}(0)$.

Next, we show $x \in F(S)$. Notice that

$$
\left\|x_{n}-S_{n} y_{n}\right\| \leq\left\|x_{n}-x_{n+1}\right\|+\alpha_{n}\left\|f\left(x_{n}\right)-S_{n} y_{n}\right\|+\beta_{n}\left\|x_{n}-S_{n} y_{n}\right\| .
$$

By using (3.3) one has

$$
\lim _{n \rightarrow \infty}\left\|x_{n}-S_{n} y_{n}\right\|=0 .
$$


It follows that

$$
\left\|S_{n} x_{n}-x_{n}\right\| \leq\left\|x_{n}-z_{n}\right\|+\left\|z_{n}-y_{n}\right\|+\left\|S_{n} y_{n}-x_{n}\right\| .
$$

By using (3.4), (3.6) and (3.7), one has

$$
\lim _{n \rightarrow \infty}\left\|x_{n}-S_{n} x_{n}\right\|=0 .
$$

Notice that

$$
\begin{aligned}
\left\|S x_{n}-x_{n}\right\| & \leq\left\|S x_{n}-\left(\delta_{n} x_{n}+\left(1-\delta_{n}\right) S x_{n}\right)\right\|+\left\|S_{n} x_{n}-x_{n}\right\| \\
& \leq \delta_{n}\left\|S x_{n}-x_{n}\right\|+\left\|S_{n} x_{n}-x_{n}\right\| .
\end{aligned}
$$

This implies from (3.8), one has

$$
\lim _{n \rightarrow \infty}\left\|x_{n}-S x_{n}\right\|=0 .
$$

By using Lemma 2.2 , we find $x \in F(S)$. This completes the proof that $x \in \Omega$. It follows that

$$
\limsup _{n \rightarrow \infty}\left\langle f(q)-q, x_{n}-q\right\rangle \leq 0 .
$$

This implies from 3.3 that

$$
\limsup _{n \rightarrow \infty}\left\langle f(q)-q, x_{n+1}-q\right\rangle \leq 0
$$

Notice that

$$
\begin{aligned}
\left\|x_{n+1}-q\right\|^{2} \leq & \alpha_{n}\left\langle f\left(x_{n}\right)-q, x_{n+1}-q\right\rangle+\beta_{n}\left\|x_{n}-q\right\|\left\|x_{n+1}-q\right\|+\gamma_{n}\left\|S_{n} y_{n}-q\right\|\left\|x_{n+1}-q\right\| \\
\leq & \alpha_{n}\left\langle f\left(x_{n}\right)-f(q), x_{n+1}-q\right\rangle+\alpha_{n}\left\langle f(q)-q, x_{n+1}-q\right\rangle+\beta_{n}\left\|x_{n}-q\right\|\left\|x_{n+1}-q\right\| \\
& +\gamma_{n}\left\|\left(z_{n}-s_{n} A z_{n}+e_{n}\right)-\left(p-s_{n} A p\right)\right\|\left\|x_{n+1}-q\right\| \\
\leq & \alpha_{n}\left\|f\left(x_{n}\right)-f(q)\right\|\left\|x_{n+1}-q\right\|+\alpha_{n}\left\langle f(q)-q, x_{n+1}-q\right\rangle+\beta_{n}\left\|x_{n}-q\right\|\left\|x_{n+1}-q\right\| \\
& +\gamma_{n}\left\|z_{n}-p\right\|\left\|x_{n+1}-q\right\|+\left\|e_{n}\right\|\left\|x_{n+1}-q\right\| \\
\leq & (1-\alpha(1-\beta))\left\|x_{n}-q\right\|\left\|x_{n+1}-q\right\|+\alpha_{n}\left\langle f(q)-q, x_{n+1}-q\right\rangle+\left\|e_{n}\right\|\left\|x_{n+1}-q\right\| .
\end{aligned}
$$

It follows that

$$
\left\|x_{n+1}-q\right\|^{2} \leq\left(1-\alpha_{n}(1-\beta)\right)\left\|x_{n}-q\right\|^{2}+2 \alpha_{n}\left\langle f(q)-q, x_{n+1}-q\right\rangle+2\left\|e_{n}\right\|\left\|x_{n+1}-q\right\| .
$$

By using Lemma 2.6, we find that $\lim _{n \rightarrow \infty}\left\|x_{n}-q\right\|=0$. This completes the proof.

Remark 3.2. Let $C$ be a nonempty closed and convex subset of $H$ and $A: C \rightarrow H$ be a mapping. Recall that the classical variational inequality is to find an $x \in C$ such that

$$
\langle A x, y-x\rangle \geq 0, \quad \forall y \in C .
$$

Projection methods have been recently investigated for solving variational inequality (3.9). It is known that $x$ is a solution to $(3.9)$ iff $x$ is a fixed point of the mapping $\operatorname{Proj}_{C}(I-r A)$, where $I$ denotes the identity on $H$. If $A$ is strongly monotone and Lipschitz, then problem (3.9) has a unique solution. If $A$ is inversestrongly monotone, then $\operatorname{Proj}_{C}(I-r A)$ is nonexpansive. Moreover, if $C$ is bounded, closed and convex, then the existence of solutions of the variational inequality is guaranteed by the nonexpansivity of the mapping $\operatorname{Proj}_{C}(I-r A)$. Let $i_{C}$ be a function defined by $i_{C}(x)=0, x \in C, i_{C}(x)=\infty, x \notin C$. It is easy to see that $i_{C}$ is a proper lower and semicontinuous convex function on $H$, and the subdifferential $\partial i_{C}$ of $i_{C}$ is maximal monotone. Define the resolvent $J_{s}:=\left(I+s \partial i_{C}\right)^{-1}$ of the subdifferential operator $\partial i_{C}$. Letting $x=J_{s} y$, we find that

$$
\begin{aligned}
y \in x+s \partial i_{C} x & \Longleftrightarrow y \in x+s N_{C} x \\
& \Longleftrightarrow\langle y-x, v-x\rangle \leq 0, \forall v \in C \\
& \Longleftrightarrow x=\operatorname{Proj}_{C} y,
\end{aligned}
$$

where $N_{C} x:=\{e \in H:\langle e, v-x\rangle, \forall v \in C\}$. 
Putting $B=\partial i_{C}$ in Theorems 3.1, we find the following result.

Corollary 3.3. Let $C$ be a closed convex subset of a real Hilbert space $H$. Let $A: C \rightarrow H$ be an inversestrongly monotone mapping with the positive constant $\alpha$ and let $F$ be a bifunction from $C \times C$ to $\mathbb{R}$ which satisfies (A1)-(A4). Let $S: C \rightarrow H$ be a strictly pseudocontractive mapping with the constant $\kappa \in[0,1)$ and let $f$ be a contractive mapping on $H$ with the constant $\beta \in(0,1)$. Assume that $\Omega=F(S) \cap V I(C, A) \cap E P(F)$ is nonempty. Let $\left\{r_{n}\right\}$ and $\left\{s_{n}\right\}$ be positive real number sequences. Let $\left\{\alpha_{n}\right\},\left\{\beta_{n}\right\},\left\{\gamma_{n}\right\}$ and $\left\{\delta_{n}\right\}$ be real number sequences in $(0,1)$ such that $\alpha_{n}+\beta_{n}+\gamma_{n}=1$. Let $\left\{x_{n}\right\}$ be a sequence generated in the process: $x_{1} \in H, y_{n}=P_{C}\left(z_{n}-s_{n} A z_{n}+e_{n}\right), x_{n+1}=\alpha_{n} f\left(x_{n}\right)+\beta_{n} x_{n}+\gamma_{n} \delta_{n} y_{n}+\left(1-\delta_{n}\right) \gamma_{n} S y_{n}$, where $\left\{e_{n}\right\}$ is a sequence in $H$ and $\left\{z_{n}\right\}$ is a sequence in $C$ such that $F\left(z_{n}, z\right)+\frac{1}{r_{n}}\left\langle z-z_{n}, z_{n}-x_{n}\right\rangle \geq 0, \forall z \in C$. Assume that the control sequences satisfy the following conditions: $\lim _{n \rightarrow \infty} \alpha_{n}=0$ and $\sum_{n=1}^{\infty} \alpha_{n}=\infty$; $0<\liminf _{n \rightarrow \infty} \beta_{n} \leq \limsup _{n \rightarrow \infty} \beta_{n}<1 ; \sum_{n=1}^{\infty}\left\|e_{n}\right\|<\infty, \lim _{n \rightarrow \infty}\left|\delta_{n+1}-\delta_{n}\right|=0$ and $\kappa \leq \delta_{n} \leq \delta<1$; $\lim _{n \rightarrow \infty}\left|r_{n+1}-r_{n}\right|=0$ and $\liminf _{n \rightarrow \infty} r_{n}>0 ; \lim _{n \rightarrow \infty}\left|s_{n+1}-s_{n}\right|=0,0<s \leq s_{n} \leq s^{\prime}<2 \alpha$, where $\delta, s, s^{\prime}$ are real constants. Then $\left\{x_{n}\right\}$ converges strongly to $q \in \Omega$, which is also a unique solution to the variational inequality $\langle f(x)-x, x-y\rangle \geq 0, \forall y \in C$.

Putting $\delta_{n}=0$ and $S=I$, we have the following results.

Corollary 3.4. Let $C$ be a closed convex subset of a real Hilbert space $H$. Let $A: C \rightarrow H$ be an inversestrongly monotone mapping with the positive constant $\alpha$ and let $B$ be a maximal monotone operator on $H$. Let $F$ be a bifunction from $C \times C$ to $\mathbb{R}$ which satisfies (A1)-(A4). Let $f$ be a contractive mapping on $H$ with the constant $\beta \in(0,1)$. Assume that $\Omega=(A+B)^{-1}(0) \cap E P(F)$ is nonempty. Let $\left\{r_{n}\right\}$ and $\left\{s_{n}\right\}$ be positive real number sequences. Let $\left\{\alpha_{n}\right\},\left\{\beta_{n}\right\}$ and $\left\{\gamma_{n}\right\}$ be real number sequences in $(0,1)$ such that $\alpha_{n}+\beta_{n}+\gamma_{n}=1$. Let $\left\{x_{n}\right\}$ be a sequence generated in the process: $x_{1} \in H, y_{n}=\left(I+s_{n} B\right)^{-1}\left(z_{n}-\right.$ $\left.s_{n} A z_{n}+e_{n}\right), x_{n+1}=\alpha_{n} f\left(x_{n}\right)+\beta_{n} x_{n}+\gamma_{n} y_{n}$, where $\left\{e_{n}\right\}$ is a sequence in $H$ and $\left\{z_{n}\right\}$ is a sequence in $C$ such that $F\left(z_{n}, z\right)+\frac{1}{r_{n}}\left\langle z-z_{n}, z_{n}-x_{n}\right\rangle \geq 0, \forall z \in C$. Assume that the control sequences satisfy the following conditions: $\lim _{n \rightarrow \infty} \alpha_{n}=0$ and $\sum_{n=1}^{\infty} \alpha_{n}=\infty ; 0<\liminf _{n \rightarrow \infty} \beta_{n} \leq \limsup _{n \rightarrow \infty} \beta_{n}<1 ; \sum_{n=1}^{\infty}\left\|e_{n}\right\|<\infty$; $\lim _{n \rightarrow \infty}\left|r_{n+1}-r_{n}\right|=0$ and $\liminf _{n \rightarrow \infty} r_{n}>0$; $\lim _{n \rightarrow \infty}\left|s_{n+1}-s_{n}\right|=0,0<s \leq s_{n} \leq s^{\prime}<2 \alpha$, where $\delta, s, s^{\prime}$ are real constants. Then $\left\{x_{n}\right\}$ converges strongly to $q=P_{\Omega} f(q)$.

Corollary 3.5. Let $C$ be a closed convex subset of a real Hilbert space $H$. Let $F$ be a bifunction from $C \times C$ to $\mathbb{R}$ which satisfies (A1)-(A4). Let $S: C \rightarrow H$ be a strictly pseudocontractive mapping with the constant $\kappa \in[0,1)$ and let $f$ be a contractive mapping on $H$ with the constant $\beta \in(0,1)$. Assume that $\Omega=F(S) \cap E P(F)$ is nonempty. Let $\left\{r_{n}\right\}$ be a positive real number sequence. Let $\left\{\alpha_{n}\right\},\left\{\beta_{n}\right\},\left\{\gamma_{n}\right\}$ and $\left\{\delta_{n}\right\}$ be real number sequences in $(0,1)$ such that $\alpha_{n}+\beta_{n}+\gamma_{n}=1$. Let $\left\{x_{n}\right\}$ be a sequence generated in the process: $x_{1} \in H, x_{n+1}=\alpha_{n} f\left(x_{n}\right)+\beta_{n} x_{n}+\gamma_{n} \delta_{n} z_{n}+\left(1-\delta_{n}\right) \gamma_{n} S z_{n}$, where $\left\{z_{n}\right\}$ is a sequence in $C$ such that $F\left(z_{n}, z\right)+\frac{1}{r_{n}}\left\langle z-z_{n}, z_{n}-x_{n}\right\rangle \geq 0, \forall z \in C$. Assume that the control sequences satisfy the following conditions: $\lim _{n \rightarrow \infty} \alpha_{n}=0$ and $\sum_{n=1}^{\infty} \alpha_{n}=\infty ; 0<\liminf _{n \rightarrow \infty} \beta_{n} \leq \limsup _{n \rightarrow \infty} \beta_{n}<1 ; \lim _{n \rightarrow \infty}\left|\delta_{n+1}-\delta_{n}\right|=0$ and $\kappa \leq \delta_{n} \leq \delta<1 ; \lim _{n \rightarrow \infty}\left|r_{n+1}-r_{n}\right|=0$ and $\liminf _{n \rightarrow \infty} r_{n}>0 ;$, where $\delta$ is a real constant. Then $\left\{x_{n}\right\}$ converges strongly to $q=P_{\Omega} f(q)$.

Put $r_{n}=1$ and $F(x, y)=0$ for any $x, y \in C$. By taking the initial in $C$, we find the following result.

Corollary 3.6. Let $C$ be a closed convex subset of a real Hilbert space $H$. Let $A: C \rightarrow H$ be an inversestrongly monotone mapping with the positive constant $\alpha$ and let $B$ be a maximal monotone operator such that $D(B) \subset C$. Let $S$ be a strictly pseudocontractive mapping on $C$ with the constant $\kappa \in[0,1)$ and let $f$ be a contractive mapping on $C$ with the constant $\beta \in(0,1)$. Assume that $\Omega=F(S) \cap(A+B)^{-1}(0)$ is nonempty. Let $\left\{s_{n}\right\}$ be a positive real number sequence. Let $\left\{\alpha_{n}\right\},\left\{\beta_{n}\right\},\left\{\gamma_{n}\right\}$ and $\left\{\delta_{n}\right\}$ be real number sequences in $(0,1)$ such that $\alpha_{n}+\beta_{n}+\gamma_{n}=1$. Let $\left\{x_{n}\right\}$ be a sequence generated in the process: $x_{1} \in C$, $y_{n}=\left(I+s_{n} B\right)^{-1}\left(x_{n}-s_{n} A x_{n}+e_{n}\right), x_{n+1}=\alpha_{n} f\left(x_{n}\right)+\beta_{n} x_{n}+\gamma_{n} \delta_{n} y_{n}+\left(1-\delta_{n}\right) \gamma_{n} S y_{n}$, where $\left\{e_{n}\right\}$ is a sequence in $H$. Assume that the control sequences satisfy the following conditions: $\lim _{n \rightarrow \infty} \alpha_{n}=0$ and $\sum_{n=1}^{\infty} \alpha_{n}=\infty ; 0<\liminf _{n \rightarrow \infty} \beta_{n} \leq \limsup _{n \rightarrow \infty} \beta_{n}<1 ; \sum_{n=1}^{\infty}\left\|e_{n}\right\|<\infty, \lim _{n \rightarrow \infty}\left|\delta_{n+1}-\delta_{n}\right|=0$ and $\kappa \leq \delta_{n} \leq \delta<1 ; \lim _{n \rightarrow \infty}\left|s_{n+1}-s_{n}\right|=0,0<s \leq s_{n} \leq s^{\prime}<2 \alpha$, where $\delta, s, s^{\prime}$ are real constants. Then $\left\{x_{n}\right\}$ converges strongly to $q=P_{\Omega} f(q)$. 
Remark 3.7. Minimize the following $l_{1}$-least square problem: $\min _{x \in R^{3}}\|x\|_{1}+\frac{1}{2}\|x\|_{2}^{2}+(1,2,3) x-5$, where $x=\left(x_{1}, x_{2}, x_{3}\right)^{T}$. Let $f(x)=\frac{1}{2}\|x\|_{2}^{2}+(1,2,3) x-5$ and $B x=\|x\|_{1}$. Then $\nabla f$ is 1-inverse-strongly monotone and $\nabla=\left(x_{1}+1, x_{2}+2, x_{3}+3\right)^{T}$. Suppose that $Q \in R^{64 \times 64}$ is a positive semidefinite matrix that the maximum eigenvalue $\kappa_{\max }$ of $Q$ is positive and that $b \in R^{64}$. Define a quadratic function $f: R^{64} \rightarrow R$ by $f(x)=\frac{1}{2}\langle x, Q x\rangle+\langle b, x\rangle, \forall x \in R^{64}$. Then $\nabla f(\cdot)=Q(\cdot)+b$ is $\kappa_{\max }$-Lipschitz continuous and $\frac{1}{\kappa_{\max }}$-inversestrongly monotone. For firmly nonexpansive mappings $\left(I+\frac{\kappa}{(n+1)^{a}} B\right)^{-1}: R^{64} \rightarrow R^{64}$, applying the algorithm, we find the following method: $x_{1} \in R^{64}$ and $x_{1} \in R^{64}$, and $y_{n}=\left(I+\frac{\kappa}{(n+1)^{a}} B\right)^{-1}\left(z_{n}-\frac{\kappa}{(n+1)^{a}} A z_{n}+\frac{\kappa}{(n+1)^{a}}\right)$, $x_{n+1}=\alpha_{n} f\left(x_{n}\right)+\beta_{n} x_{n}+\gamma_{n} \delta_{n} y_{n}+\left(1-\delta_{n}\right) \gamma_{n} S y_{n}$, where $\kappa>0, a \in[1, \infty)$ and $\left\{z_{n}\right\}$ is a sequence in $C$ such that $F\left(z_{n}, z\right)+\frac{1}{r_{n}}\left\langle z-z_{n}, z_{n}-x_{n}\right\rangle \geq 0, \forall z \in C$.

\section{Acknowledgments}

The authors are grateful to the reviewers for useful suggestions which improved the contents of this article.

\section{References}

[1] J. Balooee, Iterative algorithms for solutions of generalized regularized nonconvex variational inequalities, Nonlinear Funct. Anal. Appl., 18 (2013), 127-144. 1

[2] V. Barbu, Nonlinear semigroups and differential equations in Banach spaces, Translated from the Romanian, Editura Academiei Republicii Socialiste România, Bucharest; Noordhoff International Publishing, Leiden (1976). 2.1

[3] B. A. Bin Dehaish, A. Latif, H. Bakodah, X. Qin, A viscosity splitting algorithm for solving inclusion and equilibrium problems, J. Inequal. Appl., 2015 (2015), 14 pages.1. 2.7

[4] B. A. Bin Dehaish, X. Qin, A. Latif, O. H. Bakodah, Weak and strong convergence of algorithms for the sum of two accretive operators with applications, J. Nonlinear Convex Anal., 16 (2015), 1321-1336.1.

[5] E. Blum, W. Oettli, From optimization and variational inequalities to equilibrium problems, Math. Student, 63 (1994), 123-145.2, 2.3

[6] F. E. Browder, W. V. Petryshyn, Construction of fixed points of nonlinear mappings in Hilbert space, J. Math. Anal. Appl., 20 (1967), 197-228.2, 2.2, 2.4

[7] S. Y. Cho, Generalized mixed equilibrium and fixed point problems in a Banach space, J. Nonlinear Sci. Appl., 9 (2016), 1083-1092.1

[8] S. Y. Cho, W. Li, S. M. Kang, Convergence analysis of an iterative algorithm for monotone operators, J. Inequal. Appl., 2013 (2013), 14 pages.11

[9] S. Y. Cho, X. Qin, On the strong convergence of an iterative process for asymptotically strict pseudocontractions and equilibrium problems, Appl. Math. Comput., 235 (2014), 430-438.1

[10] W. Cholamjiak, P. Cholamjiak, S. Suantai, Convergence of iterative schemes for solving fixed point problems for multi-valued nonself mappings and equilibrium problems, J. Nonlinear Sci. Appl., 8 (2015), 1245-1256.1

[11] B. S. Choudhury, S. Kundu, A viscosity type iteration by weak contraction for approximating solutions of generalized equilibrium problem, J. Nonlinear Sci. Appl., 5 (2012), 243-251.1.

[12] J. Douglas, H. H. Rachford, On the numerical solution of heat conduction problems in two and three space variables, Trans. Amer. Math. Soc., 82 (1955), 421-439.1

[13] C. S. Hwang, An infinite family nonexpansive mappings and a relaxed cocoercive mapping, Adv. Fixed Point Theory, 4 (2014), 184-198.1

[14] J. K. Kim, P. N. Anh, Y. M. Nam, Strong convergence of an extended extragradient method for equilibrium problems and fixed point problems, J. Korean Math. Soc., 49 (2012), 187-200.1]

[15] J. K. Kim, S. Y. Cho, X. Qin, Some results on generalized equilibrium problems involving strictly pseudocontractive mappings, Acta Math. Sci., 31 (2011), 2041-2057.1

[16] L. Liu, Ishikawa and Mann iterative process with errors for nonlinear strongly accretive mappings in Banach spaces, J. Math. Anal. Appl., 194 (1995), 114-125.2.6

[17] B. Liu, C. Zhang, Strong convergence theorems for equilibrium problems and quasi- $\phi$-nonexpansive mappings, Nonlinear Funct. Anal. Appl., 16 (2011), 365-385.1

[18] S. Lv, Strong convergence of a general iterative algorithm in Hilbert spaces, J. Inequal. Appl., 2013 (2013), 18 pages.11

[19] S. Lv, A new algorithm for solving nonlinear optimization problems, J. Nonlinear Funct. Anal., 2016 (2016), 13 pages.1]

[20] X. Qin, S. Y. Cho, J. K. Kim, On the weak convergence of iterative sequences for generalized equilibrium problems and strictly pseudocontractive mappings, Optimization, 61 (2012), 805-821.1. 
[21] X. Qin, S. Y. Cho, L. Wang, A regularization method for treating zero points of the sum of two monotone operators, Fixed Point Theory Appl., 2014 (2014), 10 pages.1]

[22] X. Qin, Y. Su, Approximation of a zero point of accretive operator in Banach spaces, J. Math. Anal. Appl., 329 (2007), 415-424.1

[23] T. Ram, On existence of operator solutions of generalized vector quasi-variational inequalities, Commun. Optim. Theory, 2015 (2015), 8 pages.1

[24] R. T. Rockfellar, Monotone operators and the proximal point algorithm, SIAM J. Control Optim., 14 (1976), 877-898.1

[25] T. Suzuki, Strong convergence of Krasnoselskii and Mann's type sequences for one-parameter nonexpansive semigroups without Bochner integrals, J. Math. Anal. Appl., 305 (2005), 227-239.2.5

[26] P. Tseng, A modified forward-backward splitting methods for maximal monotone mappings, SIAM. J Control Optim., 38 (2000), 431-446.1

[27] S. Wang, On fixed point and variational inclusion problems, Filomat, 29 (2015), 1409-1417.1.

[28] J. Zhao, Strong convergence theorems for equilibrium problems, fixed point problems of asymptotically nonexpansive mappings and a general system of variational inequalities, Nonliear Funct. Anal. Appl.,16 (2011), 447-464. 1.

[29] C. Zhang, J. Zhao, Q. Dong, A strong convergence theorem by a relaxed extragradient method for a general system of variational inequalities and strict pseudo-contractions, Nonlinear Funct. Anal. Appl.,16 (2011), 465-479. 1

[30] L. C. Zhao, S. S. Chang, Strong convergence theorems for equilibrium problems and fixed point problems of strict pseudo-contraction mappings, J. Nonlinear Sci. Appl., 2 (2009), 78-91.1 